\title{
To the Optimization of Quench Performance for a Large Scale REBCO Conductor
}

\author{
Rui Kang, Davide Uglietti, Yuntao Song
}

\begin{abstract}
REBCO conductors at the level of tens kiloamperes are being developed for their application in fusion magnets. One important aspect to study is their performance in the case of a quench event happens. A conductor should survive from such an event without over design; therefore, they should be properly optimized. Using a simulation model developed based on THEA code, we studied the parameters of a REBCO conductor in a fusion magnet for their influence on the quench performance, evaluated by hot spot temperature and quench detection. According to these results, the principles to the optimization of quench performance of a REBCO conductor are discussed.
\end{abstract}

Index Terms - High temperature superconductors, quench, cable-in-conduit conductor, THEA, thermal-hydraulic

\section{INTRODUCTION}

$\mathrm{T}$ HE magnet employed with high temperature superconductor (HTS) is foreseen an enabling technology for the DEMO fusion reactor, no matter this reactor is a Tokamak [1, 2], Stellarator [3] or a compact Tokamak [4, 5]. Among the practical HTS materials, the rare-earth barium copper oxide (REBCO) is yet the most attractive one, as it has the best comprehensive performance comparing with its competitors: high or moderate critical current $\left(I_{c}\right)$ at relatively high magnetic field or temperature, high tolerance against strain and stress, and REBCO tapes are selling as end products demanding no more heat treatment for manufacturing a conductor. The main drawback would be the highest price valued in $\$ / \mathrm{kA} \cdot \mathrm{m}[6]$. Another unfavorable feature comes from its geometry: REBCO wire with the practical $I_{c}$ and length can only be achieved when it is manufactured as tape with biaxial texture. As a result, the classic cable-in-conduit-conductor (CICC), which is well developed for low temperature superconductors (LTS) like $\mathrm{NbTi}$ and $\mathrm{Nb}_{3} \mathrm{Sn}$ round wires, is not directly usable for REBCO tapes with large aspect ratio [6]. EPFL-SPC has developed a $50 \mathrm{kA}$ REBCO conductor for the Central Solenoid (CS) of EU-DEMO. Tens of REBCO tapes are first stacked in two semicircular copper profiles with rectangular slots. Then they are twisted and soldered together,

R. Kang is with the Department of Engineering and Applied Physics, University of Science and Technology of China, Hefei 230026, China. (E-mail: kangrui@mail.ustc.edu.cn). Part of this work was done while he was visiting EPFL, Swiss Plasma Center, Villigen PSI, Switzerland.

D. Uglietti is with the EPFL Swiss Plasma Center, Villigen PSI, Switzerland (E-mail: davide.uglietti@psi.ch).

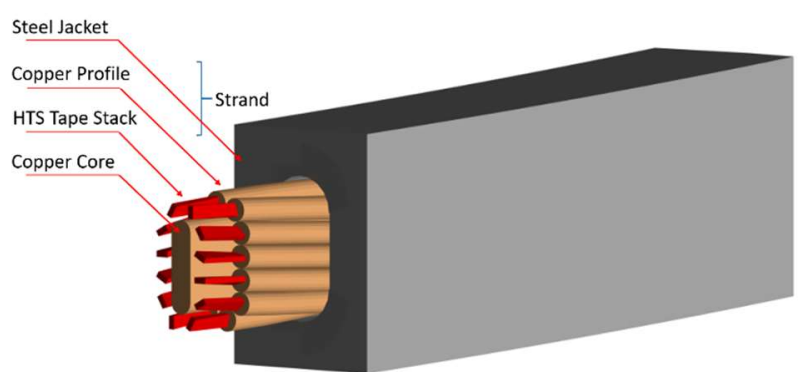

Figure 1. Sketch of the copper cored Rutherford cable conductor being developed at EPFL-SPC, from [11]. Helium is supposed to flow through the void.

becoming the so-called strand. A certain number of strands are then twisted around a copper core and jacketed with stainless steel [2]. Figure 1 is a sketch of such a conductor.

As a rather new concept, such a conductor still needs a lot of R\&D work to be fully prepared for practical use, especially in an expensive fusion reactor. One important aspect would be an optimization on its quench performance, which is regarded as a concern for all HTS conductors. Once a quench is initiated in such a conductor, the normal zone propagates rather slowly as well as the corresponding resistive voltage. As the quench might be difficult to be recognized by resistive voltage from the inductive noises, the delayed detection could eventually result into a very high hot spot temperature [7]. However, knowledge about this is relatively difficult to obtain. On one hand, quench experiments on tapes or simple coils are of limited help for understanding the behavior of a large conductor $[8,9]$. On the other hand, only few test facilities can implement a meaningful quench experiment on a large conductor [10]. As a result, the main method to access the quench performance of an HTS conductors is using specifically developed numerical codes, like THEA and 4C. Over the last few years, quench simulation of HTS conductors has processed from imitating the approach of LTS CICCs to respecting the features of HTS conductors and is giving more and more convincing results $[11,12]$. The next step would be using these tools to find the optimized parameters of an HTS conductor, as R. Heller et al have done [13].

We have developed a simulation model based on the THEA code with 1-D analysis, which can simulate the unique features

Y. Song is with the Department of Institute of Plasma Physics, Chinese Academy of Sciences, Hefei 230031, China. (E-mail: songyt@ipp.ac.cn). 
of REBCO conductors, for example the angular dependent $\mathrm{I}_{\mathrm{c}}$ against magnetic field [11, 14]. With this model, some interesting questions could be answered. A representative one is: does the angle between adjacent strands (actually the tape stacks inside) have important influence on conductor's quench behavior? The answer will decide should this angle be carefully controlled during conductor manufacturing to avoid unexpected quench performance.

In this paper, we investigate the parameters of a REBCO conductor to figure out how it should be optimized in view of its quench performance. The analysis focusses on the hot spot temperature and quench detection, the latter including normal velocity (NZPV), time from quench initiation to detection (tdec), the normal zone length needed for the detection voltage (N1_d). Those parameters are roughly divided into three groups: (1) design parameters of a conductor, including: current sharing temperature $\left(\mathrm{T}_{\mathrm{cs}}\right)$, copper current density $\left(\mathrm{J}_{\mathrm{Cu}}\right)$, copper residual resistance ratio (RRR), twist pitch of tape stack and angle of adjacent strands' orientation $\left(\theta_{\mathrm{s}}\right)$; (2) some parameters that could vary in a moderate range, including: inter-strand electrical contact resistance (ECR), n-value of REBCO tapes and magnetic field; (3) some parameters are not free to choose as they are restricted by the whole machine, including: helium mass flow rate, quench detection voltage $\left(\mathrm{V}_{\mathrm{d}}\right)$ and time constant for current dump ( $\left.\mathrm{t}_{\text {dump }}\right)$. Although some of these parameters were also studied by R. Heller [13], here they are repeated again to form a complete conclusion.

\section{Description of The Simulation Model}

The modelling of a quench in REBCO conductor is implemented by solving 1-D thermal, electrical, and hydraulic equation sets, as well as their coupling, with THEA code. Considering that the principles of the code were already well explained by the authors and some users [13, 15-17], meanwhile our REBCO model was also detailed introduced in our former publications $[11,14]$, they are only briefly recalled here.

Originated from simulating LTS cables (or conductors), 1-D analysis (as with THEA) is based on the fact that the establishment of thermal equilibrium over the cross section happens very fast. It is especially true for CICCs: (1) despite that a cable is usually composed of hundreds of LTS strands, each slim strand can be directly cooled by helium filling up the void in the bundle region with a rather high convection heat transfer coefficient; (2) at the same time, helium has much higher specific heat capacity (constant volume, $\mathrm{C}_{\mathrm{v}}$ ) than the solid materials - as compared in Figure 2 (data from [18]) - at temperature below $20 \mathrm{~K}$, which perfectly covers the critical temperature $\left(\mathrm{T}_{\mathrm{c}}\right)$ of LTS materials $(9.5 \mathrm{~K}$ for NbTi and $18 \mathrm{~K}$ for $\mathrm{Nb}_{3} \mathrm{Sn}$ ). As a result, there is unlikely temperature difference over the cable cross section and the whole cable can be regarded as one big porous wire. A 1-D thermal-hydraulic analysis can predict well its quench behavior, as was verified by the QUELL experiment $[19,20]$.

In the case of REBCO conductor, there are several different conditions: (1) the diameter of strand is about ten times higher;

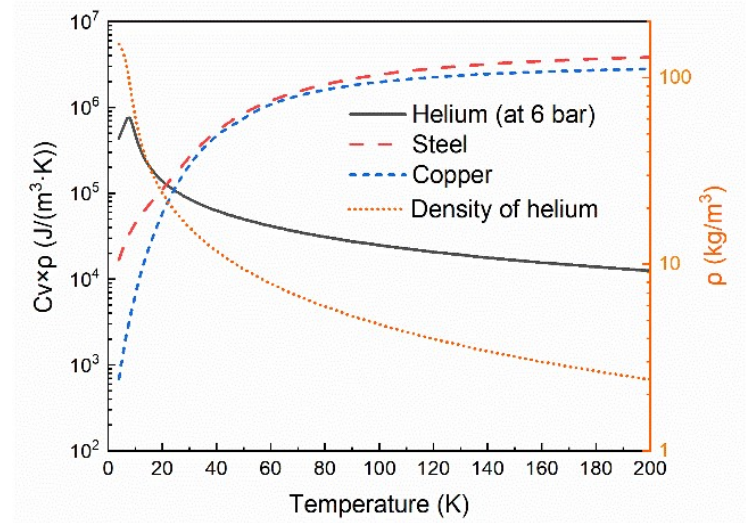

Figure 2. Heat capacity per unit volume of materials. The change of helium density is also given.

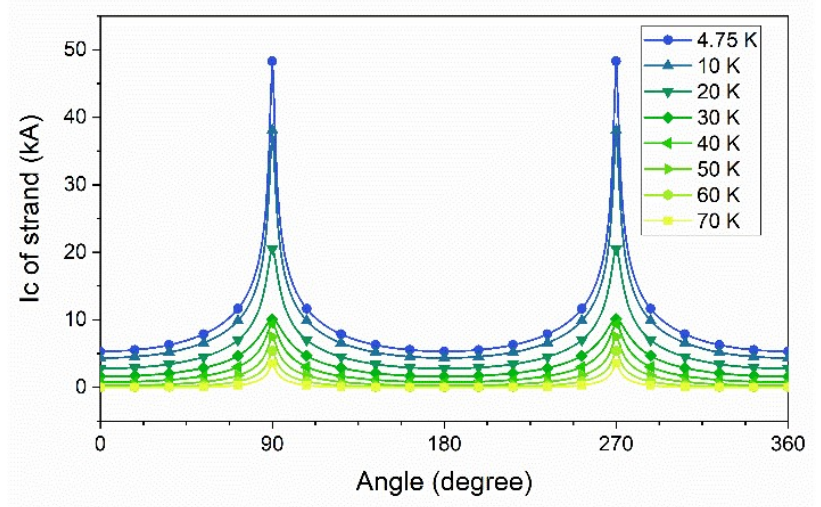

Figure 3. $\mathrm{I}_{\mathrm{c}}$ of one strand at $17.5 \mathrm{~T}$, with different $\theta$ and $\mathrm{T}$, from [11]. Note in the conductor, if two adjacent strands (stacks) have a deflection, their $\mathrm{I}_{\mathrm{c}}$ will be different at the same location.

(2) the number of strands is much reduced, but their local $I_{c}$ could be of significant difference due to the anisotropy of REBCO tape; (3) strands could still keep considerable $I_{c}$ at temperature above $20 \mathrm{~K}$ due to the high $\mathrm{T}_{\mathrm{c}}$. The first difference could make the heat transfer between helium and strands (and the tapes inside) not efficient. The second one makes that even the temperature of strands being the same, their joule heating might be different. Whereas due to the last fact, the minimum quench energy could lead to a high temperature, at which the heat capacity of solid materials starts to exceed that of helium. The last fact was also discussed by S. Hahn [21], who suggested to use "thermal runaway temperature" rather than "current sharing temperature" for the analysis of HTS quench since the difference of these two for HTS are much larger than for LTS .

Based on the above facts, we have developed a multimonolithic-strands model, evolving thermal, electrical, and hydraulic analysis to study the quench of REBCO conductor. Instead of thinking the whole cable as one element, the assumption of uniform properties is only applied on each strand. Such assumption was numerically proved to be reasonable for a soldered strand [11]. Figure 3 gives an example of the distribution of $\mathrm{I}_{\mathrm{c}}$ of each strand used in the simulation as function of the angle between magnet field and the normal direction of REBCO tape. The detailed scaling approach of $\mathrm{I}_{\mathrm{c}}$ as function of temperature, magnetic field and the angle can be 
Table 1. Parameters of the REBCO conductor

\begin{tabular}{|c|c|c|}
\hline Parameter & $\begin{array}{l}\text { Reference } \\
\text { value }\end{array}$ & $\begin{array}{l}\text { Varying } \\
\text { range }\end{array}$ \\
\hline Operating temperature $(\mathrm{K})$ & 4.75 & No \\
\hline Operating current (kA) & 50.9 & No \\
\hline Maximum magnetic field $(\mathrm{T})$ & 17.5 & $5-17.5$ \\
\hline Current sharing temperature $(\mathrm{K})$ & $\sim 10.5$ & $6.5-20$ \\
\hline $\begin{array}{l}\text { Ratio of operating current to (minimum) } \\
\text { critical current }\end{array}$ & 0.8 & $0.35-0.95$ \\
\hline Number of strands & 12 & No \\
\hline Strand diameter $(\mathrm{mm})$ & 7.2 & No \\
\hline Number of REBCO tapes in each strand & 39 & Follows \\
\hline Tape width (mm) & 4 & No \\
\hline Tape thickness (mm) & 0.095 & No \\
\hline $\begin{array}{l}\text { Fraction of tape composition: REBCO: } \mathrm{Cu} \text { : } \\
\text { Hastelloy: Ag }\end{array}$ & $1: 40: 50: 4$ & No \\
\hline Copper area in each strand $\left(\mathrm{mm}^{2}\right)$ & 32 & $32-53$ \\
\hline Total cross section of one strand $\left(\mathrm{mm}^{2}\right)$ & 40.7 & Follows \\
\hline Copper area in core $\left(\mathrm{mm}^{2}\right)$ & 189 & $95-760$ \\
\hline Copper current density $\left(\mathrm{A} / \mathrm{mm}^{2}\right)$ & 89 & $62-147$ \\
\hline RRR of copper & 30 & $30-500$ \\
\hline Twist pitch of tape stack (m) & 0.4 & $0.2-10$ \\
\hline angle of adjacent strands' orientation $\left(^{\circ}\right)$ & 30 & $0-90$ \\
\hline $\begin{array}{l}\text { Inter-strand electrical contact resistance } \\
(\Omega \cdot \mathrm{m})\end{array}$ & $2 \times 10^{-6}$ & $\begin{array}{l}2 \times 10^{-\gamma}- \\
2 \times 10^{-2}\end{array}$ \\
\hline Steel area $\left(\mathrm{mm}^{2}\right)$ & 2588 & No \\
\hline Helium area $\left(\mathrm{mm}^{2}\right)$ & 151 & No \\
\hline Hydraulic diameter (mm) & 1.52 & No \\
\hline Helium inlet pressure (bar) & 6 & No \\
\hline Mass flow rate of helium (g/s) & 5 & $1-8$ \\
\hline Critical electric field (V/m) & $1 \times 10^{-4}$ & No \\
\hline n-value (n-index) & 40 & $20-60$ \\
\hline $\begin{array}{l}\text { Voltage threshold for quench detection } \\
(\mathrm{mV})\end{array}$ & 100 & $10-500$ \\
\hline Time constant for current dump (s) & 20 & $5-35$ \\
\hline Conductor length (m) & 150 & No \\
\hline
\end{tabular}

found in [14].

The quench process in this study is divided into four steps. First it is initiated by the minimum quench energy (MQE), which is determined by a try-and-error approach, corresponding to a disturbance with $1 \mathrm{~cm}$ and $1 \mathrm{~ms}$. The energy is deposited into one strand at the region with lowest $\mathrm{J}_{\mathrm{c}}(74 \mathrm{~m}$ of the total $150 \mathrm{~m}$ in this case). Then the quench is developed freely until the resistive voltage exceeds the detection threshold value $\left(\mathrm{V}_{\mathrm{d}}\right)$. After detection, 1.1s is waited for quench validation and quench protection system operation. Finally, dump the current in conductor exponentially with a time constant $t_{\text {dump }}$ and the magnetic field follows. A detailed description of the quench process is presented in [11]. In this paper, to evaluate the quench performance, the maximum hot spot temperatures $\left(\mathrm{T}_{\max }\right.$ ) of strands (the highest of the 12, in most cases they are very close, also see [11]), copper core, helium and jacket are presented in next section for comparison. NZPV, tdec, and NL_d are also presented to better understand the quench behavior. MQE is only given for reference. As this paper focus on quench performance, only $\mathrm{T}_{\max }, \mathrm{NZPV}$, tdec and NL_d are discussed. MQE, which is a measurement of conductor's stability, is only given as an input parameter, but not discussed. The parameters concerning quench modelling of the REBCO conductor are listed in Table 1. For the parameters to study, both reference values and the varying ranges are given. Some parameters will follow others when the latter change. In the below results, any parameter takes the reference value unless it is the one for study.

\section{RESULTS AND DISCUSSION}

\section{A. Design parameters of conductor}

1) Current sharing temperature

$\mathrm{T}_{\mathrm{cs}}$ at the region with highest magnetic field, or temperature margin, is an important design parameter, at least for LTS conductors [22]. In the case of LTS conductors, the motivation for a sufficiently high $\mathrm{T}_{\mathrm{cs}}$ is to ensure the stability. On the other hand, the higher $\mathrm{T}_{\mathrm{cs}}$ is, the more inefficient usage of the superconductor, since $T_{\text {cs }}$ is directly related to the amount of superconducting wires in the conductor. For HTS, stability would not be an issue, but the quench behavior might also be influenced by $\mathrm{T}_{\mathrm{cs}}$.

Figure 4 shows the $\mathrm{T}_{\max }$ of the four components of the HTS conductor. The notable difference among them was discussed in [14] and ascribed to the relatively poor transverse heat transfer. Apparently, higher $T_{c s}$ is not beneficial to the quench
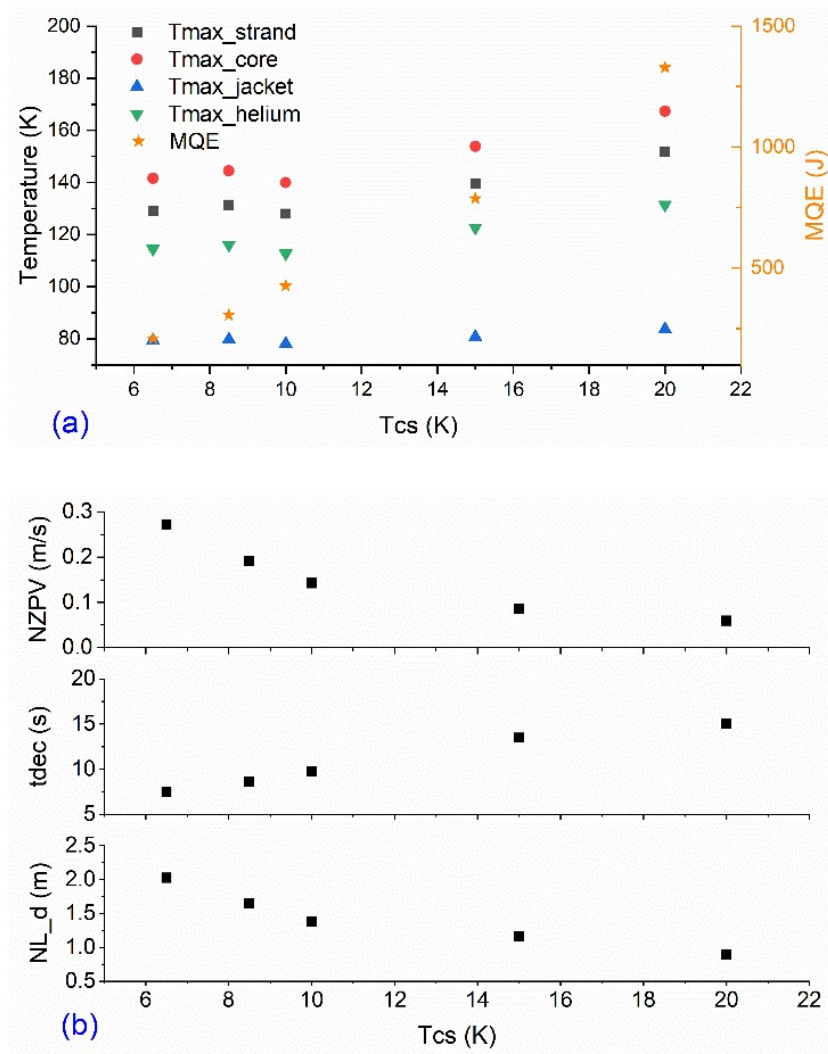

Figure 4 Quench behavior of the conductor as function of $T_{\mathrm{cs}}$. 
behavior of HTS conductor at all. Although higher $\mathrm{T}_{\mathrm{cs}}$ brings more available $I_{c}$ and postpones the heat generation, it also slows down the normal zone propagation and quench detection. Increasing $\mathrm{T}_{\mathrm{cs}}$ from $10 \mathrm{~K}$ to $20 \mathrm{~K}$, the latter effect becomes dominant and $\mathrm{T}_{\max }$ is increased by about $30 \mathrm{~K}$.

There is another thing could be noted from NL_d. For higher $\mathrm{T}_{\mathrm{cs}}$, the normal zone length needed for detection is also shorter. This is because the electrical resistance of copper is increasing with temperature significantly above $20 \mathrm{~K}$. Such situation is quite different with LTS conductor, in which NZPV is much faster, so a quench can be detected more earlier. Consequently, temperature is usually much lower when a quench is detected. Therefore, the length of normal zone is the major contribution to the resistive voltage.

From these results, high $\mathrm{T}_{\mathrm{cs}}$ would not be preferred concerning quench protection of HTS conductors.

2) Copper current density

Initially, copper was introduced to LTS wires as stabilizer: without it LTS couldn't work [23]. In CICCs, additional copper wires are also cabled together with superconducting wires for quench protection, which further reduces the quench current density and heat generation. For HTS conductors, the latter function is more likely why copper should be employed since HTS conductors are very stable. In this sense, copper in HTS conductors shouldn't be called "stabilizer". Instead, "quench protection material", or "quench protector" would be better names.

Whatever the name, it can be foreseen the importance of copper on quench behavior, which is however a contradiction. On one hand, as mentioned above, more copper in a conductor would release the heat generation during a quench. On the other hand, with same current and normal zone length, the voltage is lower due to the lower resistance, which means a slower quench detection.

Figure 5 shows the quench behavior of the HTS conductor with different $\mathrm{J}_{\mathrm{Cu}}$. From the results of $\mathrm{T}_{\max }$, one can conclude that, at least for this HTS conductor, more copper is preferred for quench protection. The effect of heat generation dominates the quench behavior instead of normal zone propagation. Twice the copper cross section from the reference case, hot spot temperature can be reduced by more than $70 \mathrm{~K}$, though quench would be detected later for more than $10 \mathrm{~s}$. Although more copper reduces the current density of the whole conductor, for conductors in CS coils in Tokamaks, it would be less a problem. The main negative factor for conductor current density comes from the large amount of steel needed to protect the conductor from hoop stress. Double copper cross section from the reference value only decrease the current density for about $16 \%$.

It should be noted that, in the HTS conductor studied in this paper, there are basically two types of copper, which is similar as in the case of LTS CICCs. One is the copper in the strand, i.e. from the tape and profile. The other type is the copper core. Their difference is the ease of current sharing: current must go through higher resistance in the latter case. With the reference parameters, these two types of copper play very similar roles. Therefore, if more copper is needed, it could be added wherever is convenient.
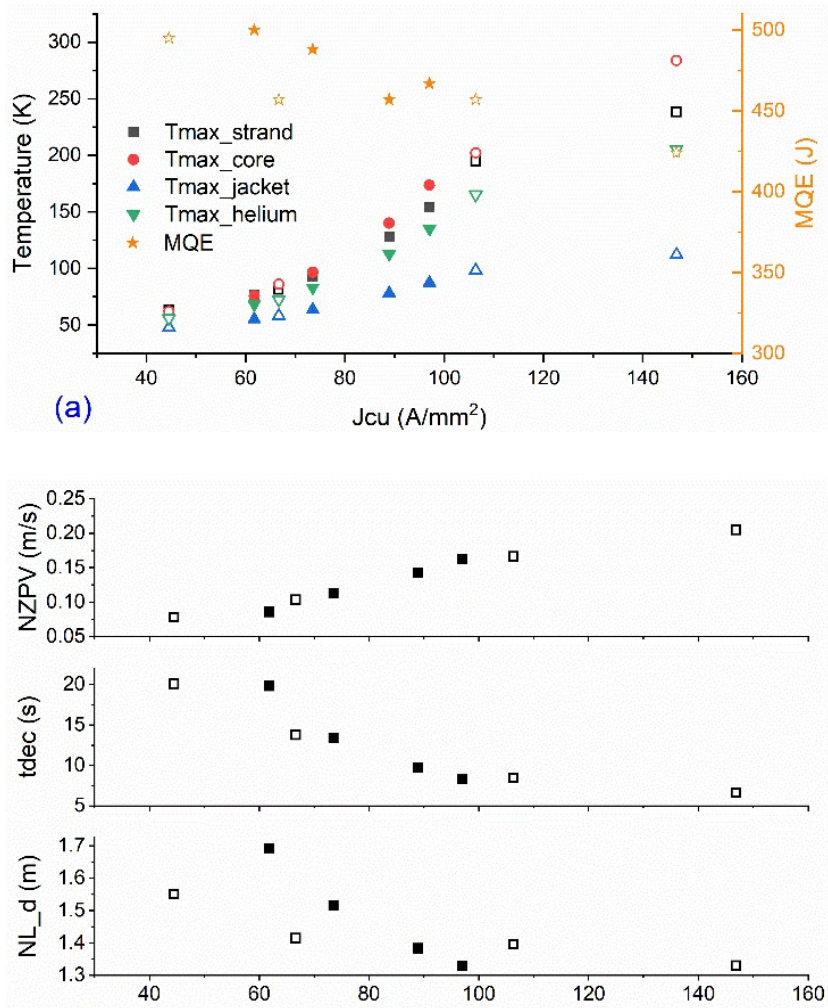

(b)

$\mathrm{Jcu}\left(\mathrm{A} / \mathrm{mm}^{2}\right)$

Figure 5. Quench behavior of the conductor as function of $\mathrm{J}_{\mathrm{Cu}}$. Solids symbols are for varying copper in strand, open symbols are for varying copper in core.
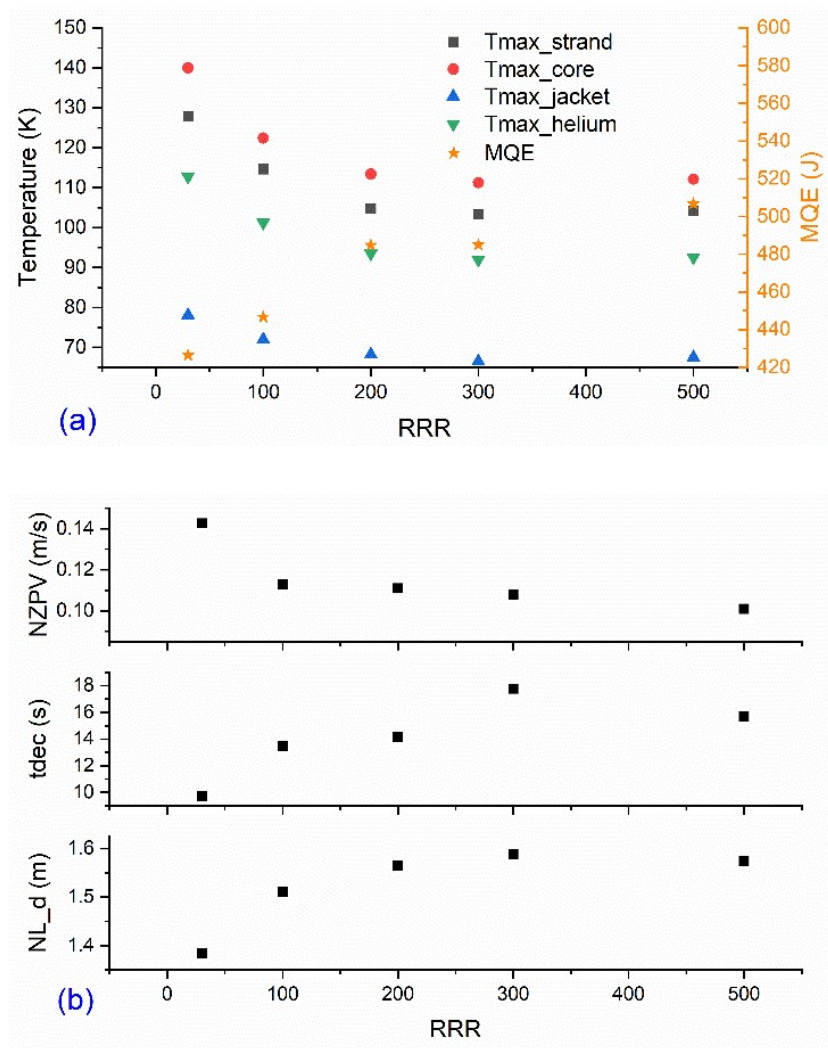

Figure 6. Quench behavior of the conductor as function of RRR. 


\section{3) Residual Resistance Ratio}

The resistivity of copper at low temperature also depends on its RRR, so RRR could also influence the quench behavior, as figure 6 shows. $T_{\max }$ decreases with RRR increasing, but the effect is limited, NZPV, tdec and NL_d as well. When RRR is higher than 300 , increase it further doesn't help a lot. Changing RRR from 30, which is the reference value, to 300 (or 500, nearly the same), $T_{\max }$ decreases by about $30 \mathrm{~K}$. Anyway, RRR must be considered together with the copper amount, as well as the total expense.

4) Twist pitch and $\theta_{\mathrm{s}}$

These two parameters are unique for REBCO conductors with twisted stacked tapes, as shown in Figure 7. The orientation of one strand is actually the one of the REBCO stack inside, as explained above. REBCO tapes have anisotropic $I_{c}$ with respect to magnetic field. Twisting it results into nonuniform $\mathrm{I}_{\mathrm{c}}$ along longitudinal direction, which is also a function of the twist pitch. The tapes in each strand have the same orientation, i.e. the same $I_{c}$, but those in different ones could not. Therefore, a question is, does $\theta_{\mathrm{s}}$ have important influence on quench behavior of the conductor? A series combination of twist pitch and $\theta_{\mathrm{s}}$ are considered to answer it. Note that in a real conductor, twisting happens both at the stack and cable level. In this simulation, the twist pitch is regarded as the total effect of these two. Although in the flat part twist of cable is not really happening, it is regarded a minor effect and is not discussed in this simulation for simplicity.

Figure 8 shows the quench behavior of the HTS conductor with respect to twist pitch, considering $\theta_{\mathrm{s}}$ is $30^{\circ}$, with which the orientation of 12 strands composes $360^{\circ}$. Increasing the twist pitch from $0.2 \mathrm{~m}$ to $1 \mathrm{~m}, \mathrm{~T}_{\max }$ reduces by about $10 \mathrm{~K}$. If the twist pitch is longer, $T_{\max }$ starts to increase. The maximum, which is about $40 \mathrm{~K}$ higher than the minimum, is achieved with a twist pitch of $7.5 \mathrm{~m}$. Such results indicate that there would be a twist pitch, with which a minimum $T_{\max }$ can be achieved. Figure 8(b) shows the parameters concerning quench propagation.

The fundamental reason why twist pitch plays a role on quench performance is, it decides the length of the $I_{c}$ peak in one strand. The longitudinal heat transfer in the conductor mainly depends on the thermal conductance of materials and mass flow rate of forced helium, which doesn't change with twist pitch. Taking one strand for example, when twist pitch is rather short $(<1 \mathrm{~m})$, heat flux can pass through the $\mathrm{I}_{\mathrm{c}}$ peak very easily, so normal zone propagation velocity is hardly blocked by this peak. On the contrary, if twist pitch is very long $(>5 \mathrm{~m}$, almost equivalent to a non-twisted cable), the normal zone will stay in $1 / 4$ twist pitch almost for the whole quench process. If twist pitch is in a moderate range, the normal zone will have to go across one wide $I_{c}$ peak. When other strands are also taken into account, the situation is more complicated. While one strand is at the $I_{c}$ valley, others are either on the hillside or the peak, as is shown in Figure 9. The ultimate result is what presented in Figure 8.

Figure 10 shows the quench behavior of the HTS conductor with different $\theta_{\mathrm{s}}$. The twist pitch is fixed as $0.4 \mathrm{~m}$. It can be seen that $T_{\max }$ hardly changes with different $\theta_{\mathrm{s}}$ in the range of $0-90^{\circ}$ : the variation is less than $2 \mathrm{~K}$ and the change of tdec is also less

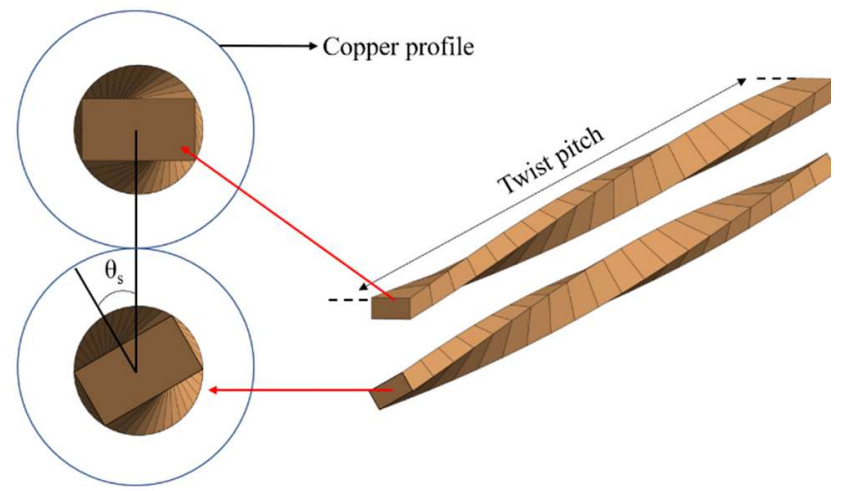

Figure 7. Sketch of the twist pitch of a strand and the angle of adjacent strands' orientation.

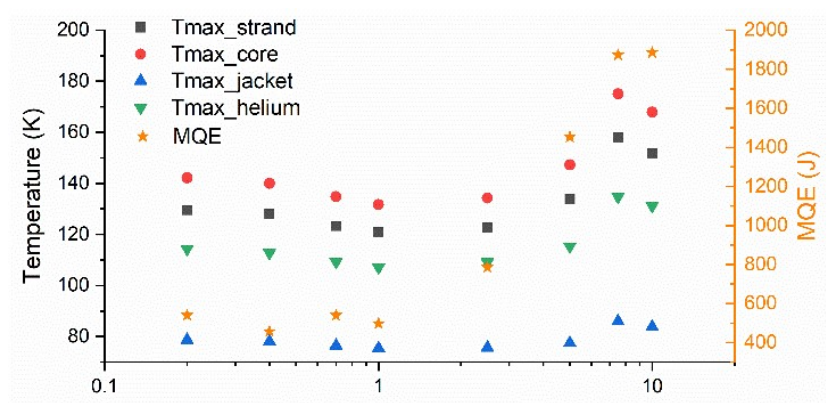

(a)

twist pitch (m)

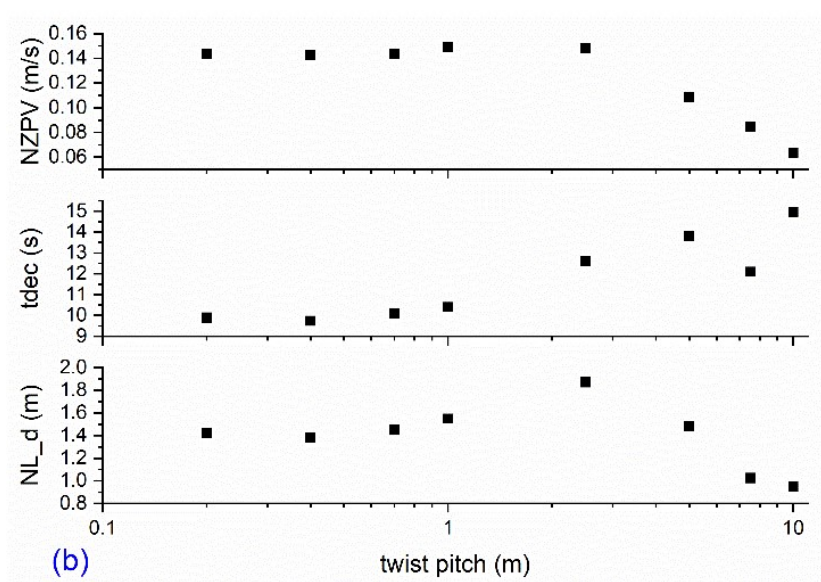

Figure 8. Quench behavior of the conductor as function of twist pitch. 


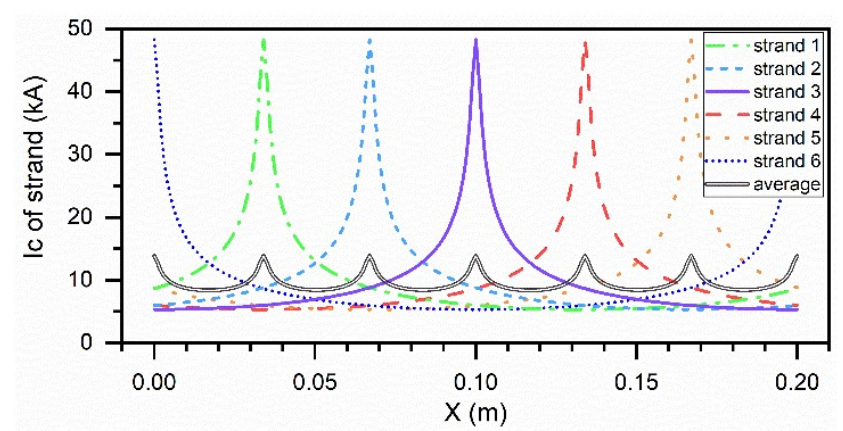

Figure 9. The change of $I_{c}$ in half twist pitch of strands. Twist pitch is $0.4 \mathrm{~m}$, and $\theta_{\mathrm{s}}$ is $30^{\circ}$. $\mathrm{I}_{\mathrm{c}}$ of the other 6 strands duplicate with these 6 . The average $I_{c}$ of one strand also shows a periodic peak.
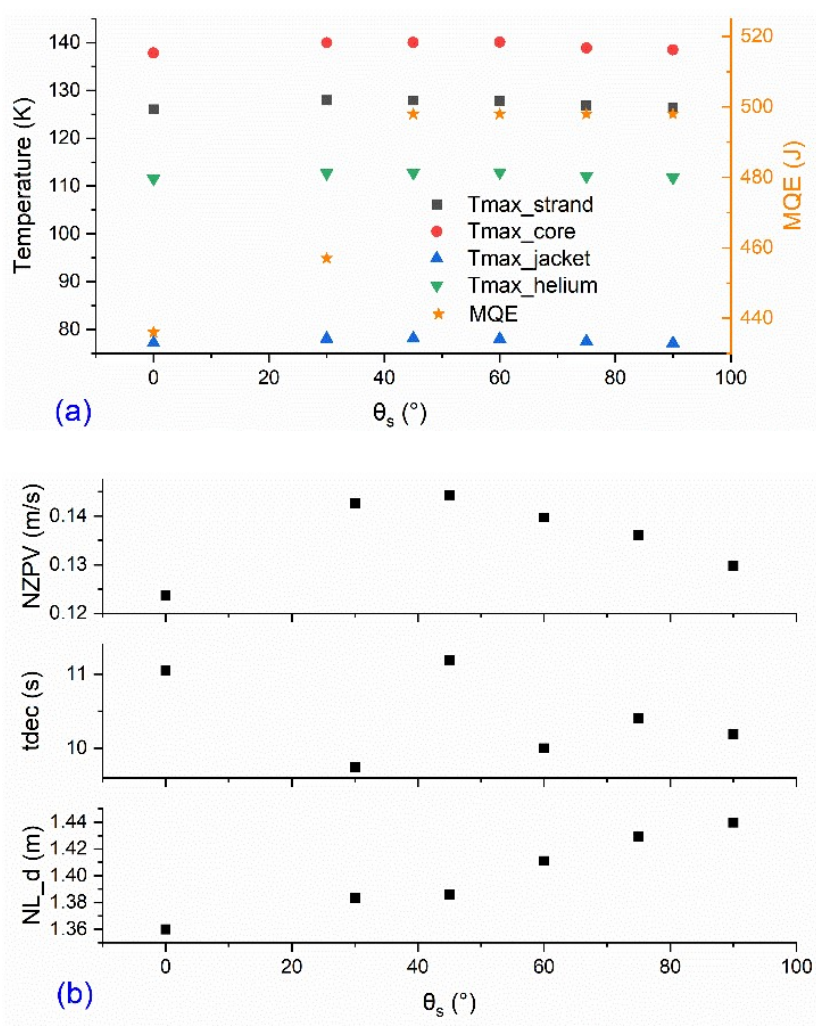

Figure 10. Quench behavior of the conductor as function of $\theta_{\mathrm{s}}$.

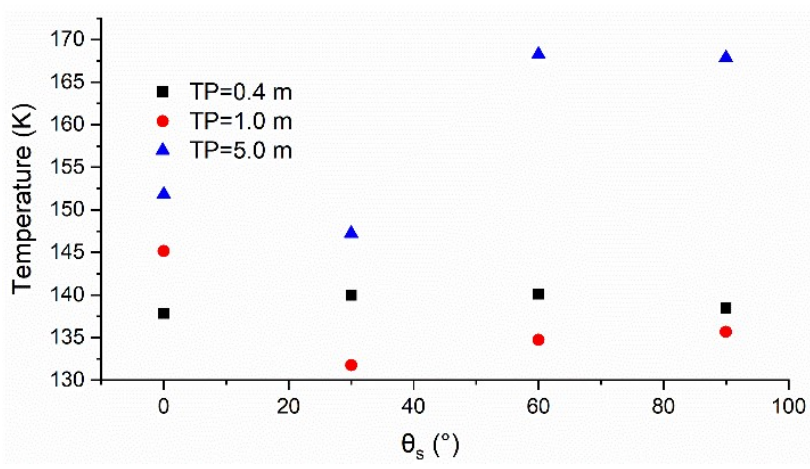

Figure 11. Quench behavior of the conductor as function of $\theta_{\mathrm{s}}$ at different twist pitch.

than $1 \mathrm{~s}$. This means that $\theta_{\mathrm{s}}$ can be left it be, which are good news for cable manufacturing.

On the other hand, the influence of $\theta_{\mathrm{s}}$ on quench behavior of the conductor is related to the twist pitch, as is shown in Figure 11. In Figure 11, the quench behavior as function of $\theta_{\mathrm{s}}$, is shown with three different twist pitches, representing respectively short $(0.4 \mathrm{~m})$, moderate $(1 \mathrm{~m})$ and long $(5 \mathrm{~m})$ twist pitch. With short twist pitch, as already discussed above, $\theta_{\mathrm{s}}$ has little influence on quench behavior. When twist pitch goes to moderate, the maximum difference is up to about $15 \mathrm{~K}$. Such difference increases to above $20 \mathrm{~K}$ when twist pitch is longer. Summarizing the above results, there are two ways to deal with twist pitch and $\theta_{\mathrm{s}}$. One is to choose certain values for them, $1 \mathrm{~m}$ and $30^{\circ}$ respectively in this case, to have a minimum $\mathrm{T}_{\max }$. Another one is to keep a short twist pitch, for example $0.4 \mathrm{~m}$, to avoid uncertainty of quench behavior due to the $\theta_{\mathrm{s}}$, which can release the conductor manufacture.

The reason why twist pitch and $\theta_{\mathrm{s}}$ can affect the quench behavior, is because they decide the $I_{c}$ variation along the conductor length. In view of this, the electrical contact resistance (ECR) between strands, as well as between strand and the copper core, would also be especially important for the twisted REBCO conductor, since it decides the ease of current bypass among strands. If ECR is too high, current in the quenched strand will be difficult to transfer to the neighbor strands, who could still have spare $\mathrm{I}_{\mathrm{c}}$.

\section{B. Varying parameters}

1) Inter-strand electrical contact resistance

Although there are some methods to change the inter-strand ECR, as in the case of LTS conductors, it is difficult to precisely control it, since it depends on too many parameters: temperature, contact pressure, roughness and so on. In view of this, a wide range of ECR is considered for the influence on quench behavior, with the reference twist pitch and $\theta_{\mathrm{s}}$, as shown in Figure 12. When ECR is varying within $2 \times 10^{-8}-2 \times 10^{-5} \Omega \cdot \mathrm{m}$, $\mathrm{T}_{\max }$ hardly changes, despite lower ECR delays quench detection. That is, when ECR is increasing in this range, on one hand, current is easier to transfer from the normal part of one strand to the neighbors which can offer more space for superconducting current, so heat generation is reduced in the quench process. On the other hand, as quench detection is delayed, heat generation happens in a longer time. According to the measurement of N. Bykovsky [24], ECR in such REBCO conductor is around $2 \times 10^{-5} \Omega \cdot \mathrm{m}$. If similar manufacture process is adopted as that sample, un-predictable quench behavior is unlikely to happen because of ECR. On the other hand, if ECR is intentionally enhanced to reduce the coupling loss, $T_{\max }$ will significantly increase averagely $35 \mathrm{~K}$ for ECR increasing for one order of magnitude. Besides, the maximum temperature gradually moves from copper core to strands, which indicates that current is locked in each strand and the copper core doesn't help reduce heat generation anymore. Therefore, if the strands are plated with high resistance layer to reduce coupling loss, it would be better to increase the copper cross section in each strand, instead of using a thick copper core.

2) n-value

Another parameter that is rather random is the n-value of the 
tapes, which could differ with the manufacturer, cabling process, temperature, magnetic field and so on. As for quench behavior, n-value will decide both quench detection and heat generation. A very high n-value means the HTS tapes, as well as the total cable, will have neglectable resistance/voltage so long as the temperature is lower than $\mathrm{T}_{\mathrm{cs}}$. However, once $\mathrm{T}_{\mathrm{cs}}$ is exceed, higher n-value results into both higher resistance and voltage signals. Such complex phenomenon can also only be studied by simulation.

Figure 13 shows the quench behavior with different n-values. The relations of $T_{\max }, \mathrm{NZPV}$ and $n$-value are quite simple. The higher is the n-value, the faster is normal zone propagation and the lower is the $T_{\max }$, but the influence on $T_{\max }$ is very small (less than $5 \mathrm{~K}$ changing $\mathrm{n}$-value from 20 to 60 ). Therefore, $\mathrm{n}$ -
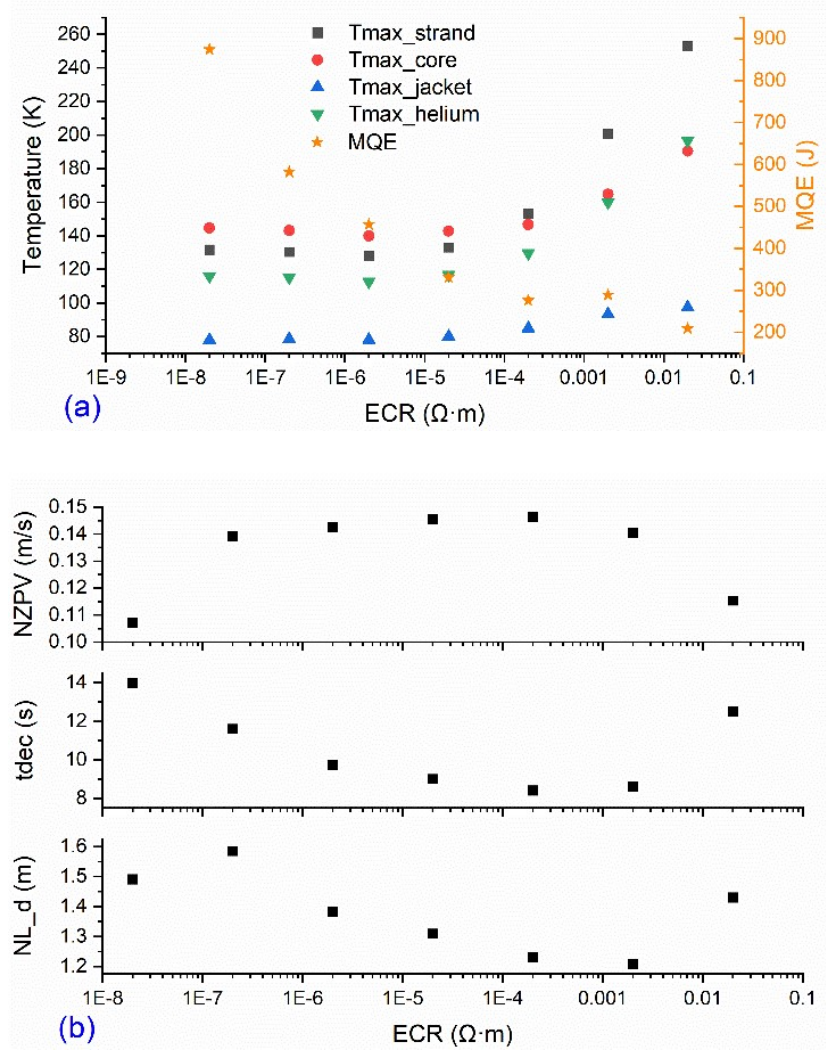

Figure 12. Quench behavior of the conductor as function of ECR.
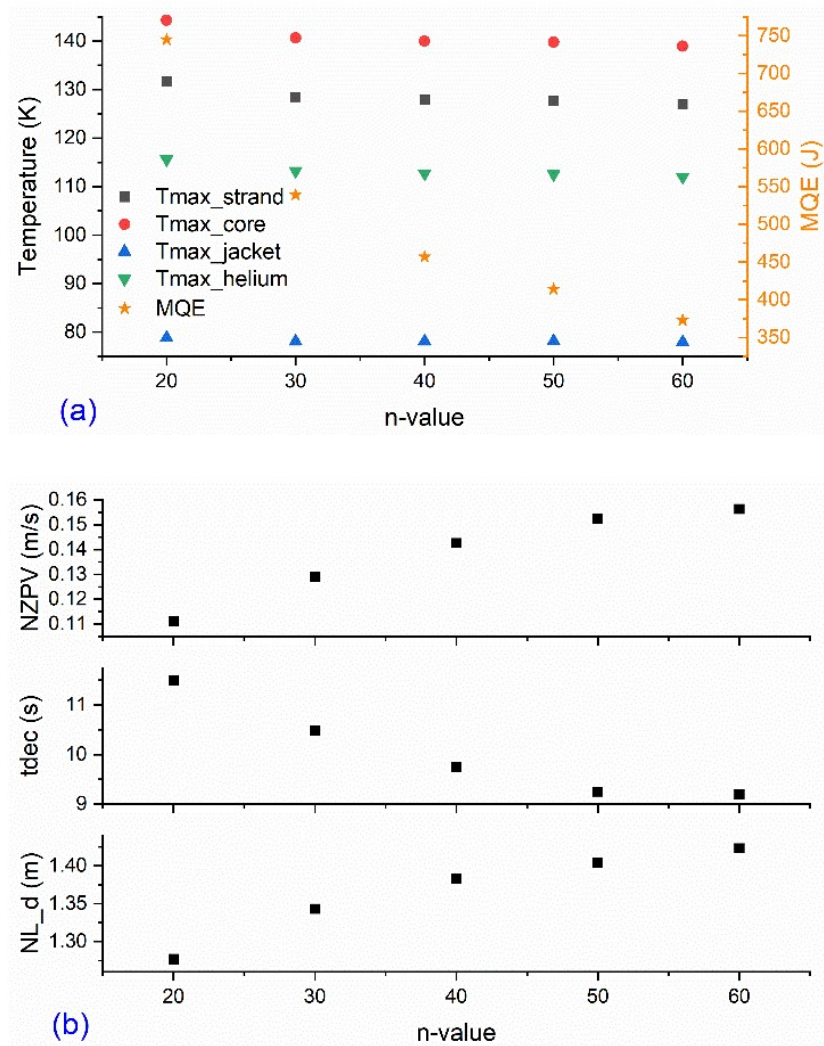

Figure 13. Quench behavior of the conductor as function of n-value. 

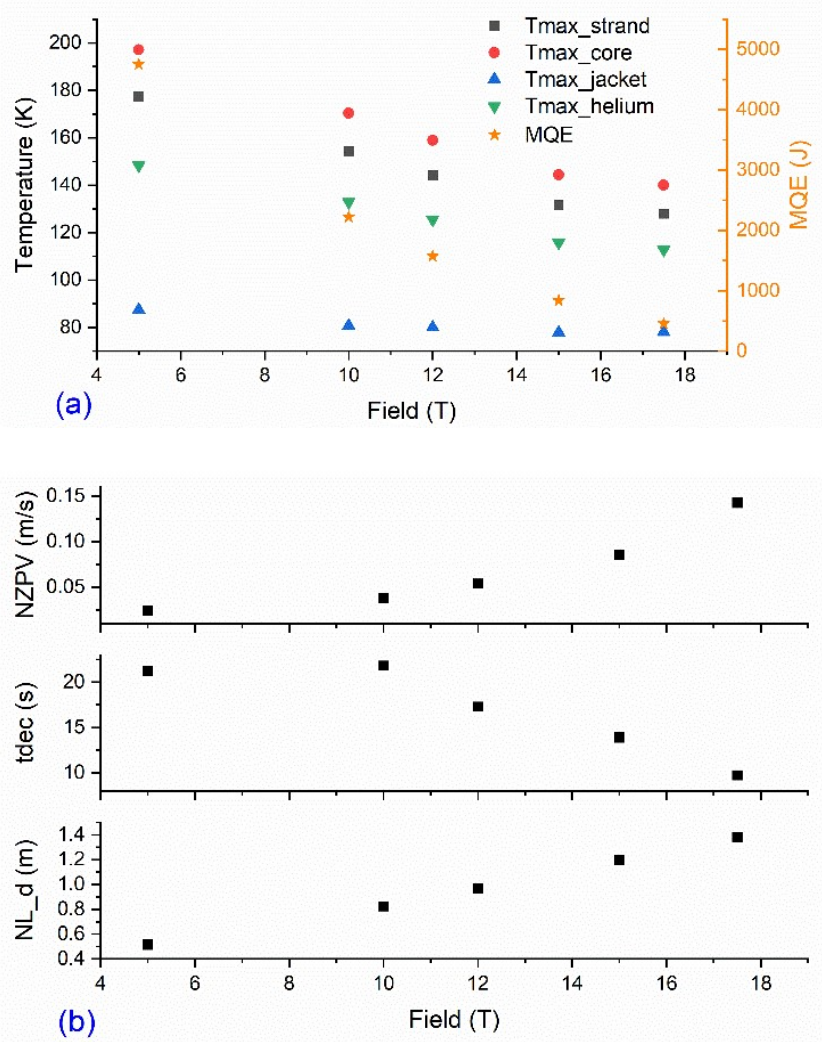

Figure 14. Quench behavior of the conductor as function of magnetic field.
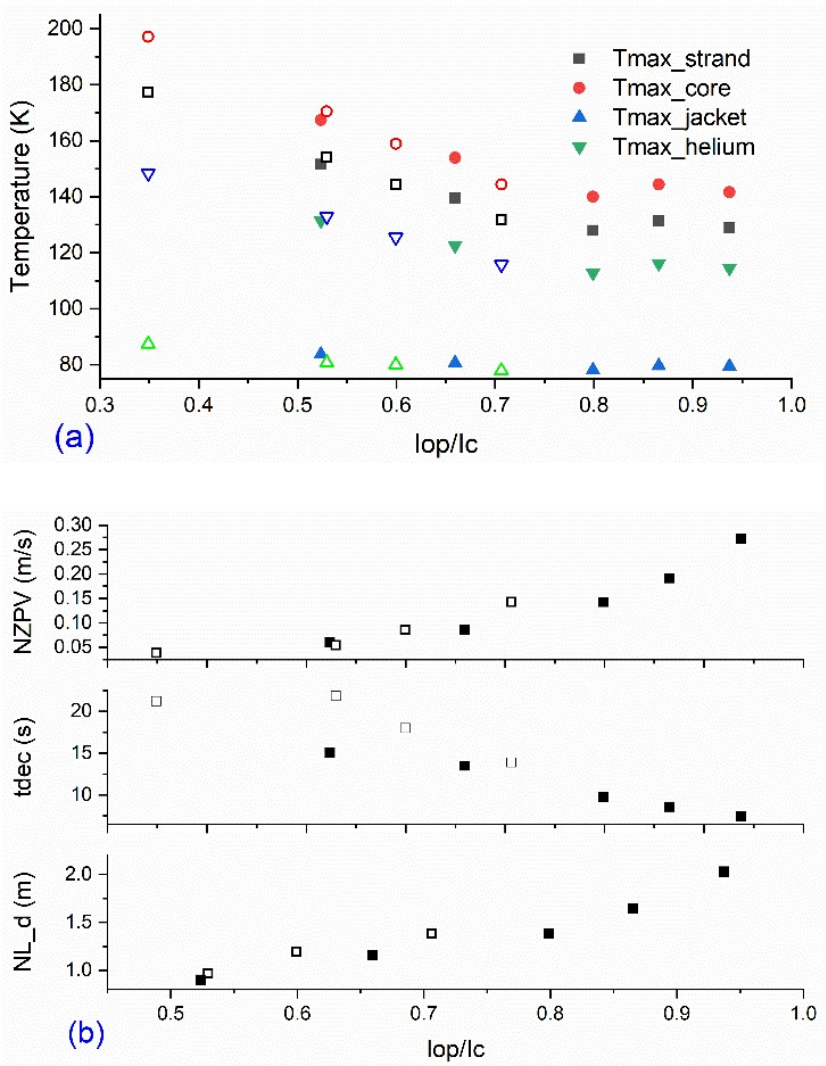

Figure 15. Quench behavior of the conductor as function of $\mathrm{I}_{\mathrm{op}} / \mathrm{I}_{\mathrm{c}}$. Solids symbols are for varying $T_{c s}$, open symbols are for varying field. value is eventually of little importance concerning quench performance for REBCO conductors.

3) Magnetic field

One more parameter that potentially has large influence on quench behavior, but is less a design parameter for the conductor, is the magnetic field, which could change in the coil winding. Figure 14 shows the quench behavior as function of magnetic field. Clearly, when quench is initiated at low field region, slower quench propagation and higher hot spot temperature would be expected, despite that much higher energy is needed to quench the conductor at low field region. In this sense, when using REBCO conductor, a graded layer winding, as is designed for Euro-DEMO CS [2], is better than pancake winding, since the former has less field variation along the conductor.

4) $\mathrm{I}_{\mathrm{op}} / \mathrm{I}_{\mathrm{c}}$

The major influence of magnetic field is the $\mathrm{I}_{\mathrm{c}}$. Lower field means higher $I_{c}$, that is lower $I_{o p} / I_{c}$, which is similar as the influence of $\mathrm{T}_{\mathrm{cs}}$. Converting the $\mathrm{x}$-axis of figure 4 and 14 into $\mathrm{I}_{\mathrm{op}} / \mathrm{I}_{\mathrm{c}}$ for these cases and put the data together, a plot of $\mathrm{T}_{\max }$ as function of $\mathrm{I}_{\mathrm{op}} / \mathrm{I}_{\mathrm{c}}$ is shown in figure 15. Interestingly, no matter how the change of $\mathrm{I}_{\mathrm{op}} / \mathrm{I}_{\mathrm{c}}$ happens, the relation between $\mathrm{T}_{\max }$ and $\mathrm{I}_{\mathrm{op}} / \mathrm{I}_{\mathrm{c}}$ appears a similar trend, especially at lower $\mathrm{I}_{\mathrm{op}} / \mathrm{I}_{\mathrm{c}}$. The same for NZPV, tdec and NL_d. Such result indicates that for HTS conductors, it is better to operate them without very large current margin, especially considering their good stability.

\section{Global parameters}

1) helium flow rate

Helium flow, which depends on the power of cryogenic system, friction in conductor and conductor length, is not totally free to choose, but is also an important parameter for quench behavior of REBCO conductor, as shown in figure 16. The relation between $T_{\max }$ and mass flow rate is very simple: $T_{\max }$ decreases as mass flow rate increases, significantly. NZPV also increases monotonically with mass flow rate. On the other hand, there is still a little trick about the effect of mass flow rate, which can be seen from the detection time and the normal zone length. With higher mass flow rate, quench is detected later with the resistive voltage, although NZPV is faster. An explanation to this interesting result is, in the case of high mass flow rate, better cooling capability lower the temperature of the cable and so does the resistance, therefore longer length is needed to reach the same resistive voltage. Although helium flow with higher speed also helps heat transfer in longitudinal direction, so higher NZPV is achieved, N1_d increases more significantly. Finally, quench is detected later. Anyway, as the price to enhance mass flow rate is increasing, it must be considered comprehensively together with many other aspects.

\section{2) Detection voltage}

In CS coil, the resistive voltage for quench detection must be distinguished from all the inductive noises. Therefore, the threshold voltage for quench detection could be limited. In the above cases, a threshold of $100 \mathrm{mV}$ is assumed. It is important to know if the real threshold is higher, how much would the $\mathrm{T}_{\max }$ increase and if it is worthy to achieve much lower 

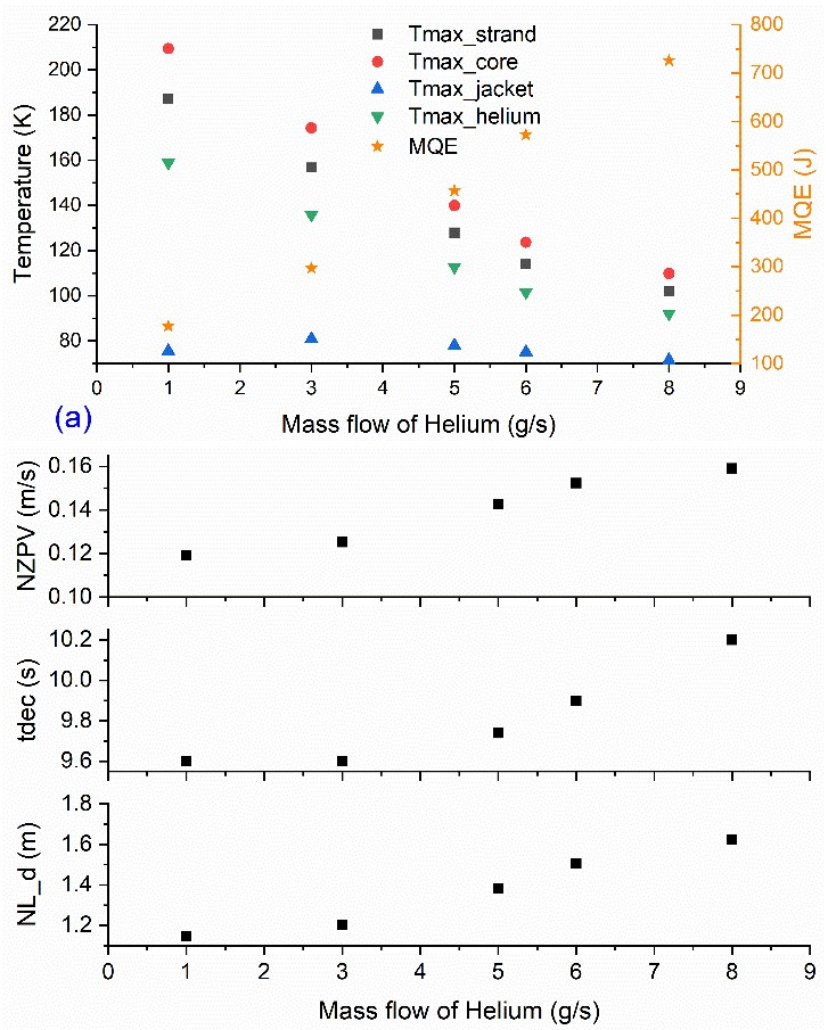

Figure 16. Quench behavior of the conductor as function of helium mass flow rate.

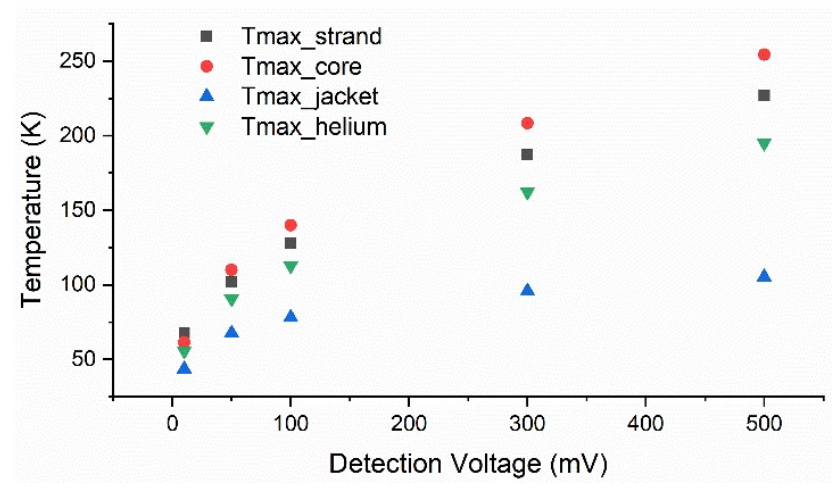

Figure 17. $\mathrm{T}_{\max }$ of strand components as function of detection voltage.

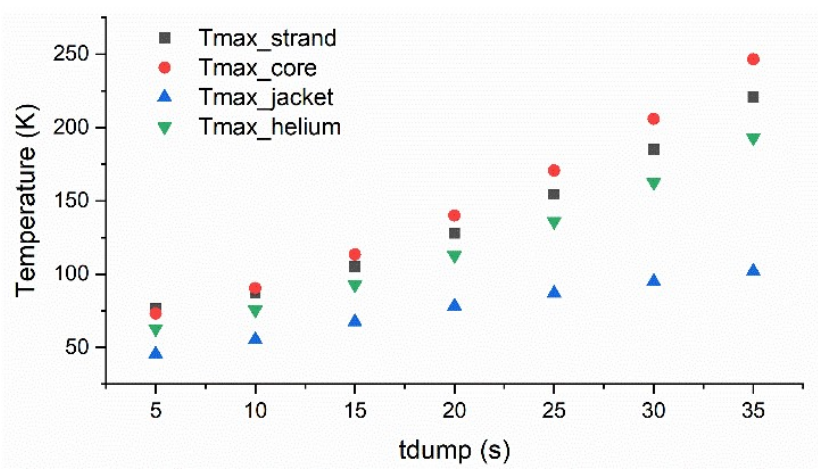

Figure 18. $\mathrm{T}_{\max }$ of strand components as function of $\mathrm{t}_{\mathrm{dump}}$ threshold. Figure 17 answers these questions. Increase the threshold from $100 \mathrm{mV}$ to $500 \mathrm{mV}, \mathrm{T}_{\max }$ will increase for more than $100 \mathrm{~K}$. In such case, other means must be adopted to inhibit $\mathrm{T}_{\max }$, like adding more copper into the conductor. On the other hand, if the threshold could be achieved as only $10 \mathrm{mV}, \mathrm{T}_{\max }$ could also be greatly reduced by about $75 \mathrm{~K}$, which leaves a lot of spaces for other design aspects, including helium flow.

\section{3) Time constant for current dump}

In above simulation, the $t_{d u m p}$ is chosen as $20 \mathrm{~s}$. This parameter is limited by the inductive voltage and the safety operation of other components, like vacuum vessel, and it also has a big influence on the quench behavior of the conductor, as shown in figure 18 . The $t_{\text {dump }}$ also has significant influence on $\mathrm{T}_{\max }$. If due to whatever reason, $\mathrm{t}_{\mathrm{dump}}$ is set as $35 \mathrm{~s}$, then again large amount of copper in the conductor or high mass flow rate of helium would be needed.

\section{CONCLUSION}

For a REBCO conductor, fast normal zone propagation doesn't always result into fast quench detection and low hot spot temperature. Therefore, when optimizing it, it is better to discuss a parameter for the influence on $\mathrm{T}_{\max }$, which is the ultimate target.

Among the design parameters for REBCO conductor, copper current density or copper cross section, as well as its RRR, has the most important influence on quench behavior. In the CS coil where hoop stress is very high and large amount of steel is needed, double copper cross section from the reference value would reduce $\mathrm{T}_{\max }$ for about $70 \mathrm{~K}$, whereas the conductor current density only decreases for about $16 \%$. Increasing RRR from 30 to $300, \mathrm{~T}_{\max }$ decreases for about $30 \mathrm{~K}$, but higher RRR hardly changes $\mathrm{T}_{\max }$. For REBCO conductor, too high $\mathrm{T}_{\mathrm{cs}}$ not only wastes the expensive HTS tape, but also worsen the quench behavior: quench detection is delayed, and hot spot temperature is raised. Although a moderate twist pitch around $1 \mathrm{~m}$ results into a lowest $\mathrm{T}_{\max }$, keep it as low as possible can avoid the influence of the angle between each two strands on quench behavior, therefore releases the cabling process.

ECR has little influence on $T_{\max }$ so long as it stays in the range of $2 \times 10^{-8}-2 \times 10^{-5} \Omega \cdot \mathrm{m}$. Further decreasing it results into an increase of $\mathrm{T}_{\max }$ for $35 \mathrm{~K}$ per orders of magnitude. The effect of the magnetic field where a quench happens, supports the degraded layer winding, since in low field region, quench detection is strongly delayed and $\mathrm{T}_{\max }$ is increased. The $\mathrm{n}$-value has very little influence. Varying from 20 to $60, T_{\max }$ only changes for about $5 \mathrm{~K}$.

Helium mass flow rate, detection voltage and $t_{\text {dump }}$ all have significant influence on quench behavior but are not free to choose. The available values (or at least ranges) of these parameters must be considered during conductor design.

\section{REFERENCE}

[1] J. G. Li and Y. X. Wan, "Present State of Chinese Magnetic Fusion Development and Future Plans," Journal of Fusion Energy, vol. 38, no. 1, pp. 113-124, Feb 2019, doi: 10.1007/s10894-018-0165-2. 
[2] R. Wesche et al., "Central solenoid winding pack design for DEMO," Fusion Eng. Des., vol. 124, pp. 82-85, Nov 2017, doi: 10.1016/j.fusengdes.2017.04.052.

[3] N. Yanagi et al., "Magnet design with 100-kA HTS STARS conductors for the helical fusion reactor," Cryogenics, vol. $\quad 80, \quad$ pp. 243-249, 2016, doi: 10.1016/j.cryogenics.2016.06.011.

[4] A. Sykes et al., "Compact fusion energy based on the spherical tokamak," Nucl. Fusion, vol. 58, no. 1, Jan 2018, doi: ARTN 016039

10.1088/1741-4326/aa8c8d.

[5] D. G. Whyte, J. Minervini, B. LaBombard, E. Marmar, L. Bromberg, and M. Greenwald, "Smaller \& Sooner: Exploiting High Magnetic Fields from New Superconductors for a More Attractive Fusion Energy Development Path," Journal of Fusion Energy, vol. 35, no. 1, pp. 41-53, 2016, doi: 10.1007/s10894-015-0050-1.

[6] D. Uglietti, "A review of commercial high temperature superconducting materials for large magnets: from wires and tapes to cables and conductors," Superconductor Science and Technology, vol. 32, no. 5, p. 053001, 2019, doi: 10.1088/13616668/ab06a2.

[7] Y. Iwasa, Case Study in Superconducting Magnets. Springer US, 2009.

[8] N. Glowa, R. Wesche, and P. Bruzzone, "Quench Studies of YBCO Insulated and Noninsulated Pancake Coils," IEEE Trans. Appl. Supercond., vol. 24, no. 3, Jun 2014, Art no. 4701105, doi: 10.1109/tasc.2013.2287636.

[9] X. J. Luo, S. Inoue, and N. Amemiya, "Quench Experiments of Conduction-Cooled Coated Conductors With Various Copper-Stabilizer Thicknesses," (in English), IEEE Trans. Appl. Supercond., vol. 30, no. 4, Jun 2020, doi: 10.1109/Tasc.2019.2960758.

[10] O. Dicuonzo et al., "Quench Experiment on 15kA HTS Cables Made of Stack of Tapes," presented at the To be presented at Applied Superconductivity Conference 2020.

[11] R. Kang, D. Uglietti, and Y. Song, "Modelling quench of a 50 kA REBCO conductor with soldered-twisted-stacked-tapecable strands," Cryogenics, vol. 106, 2020, doi: 10.1016/j.cryogenics.2020.103037.

[12] A. Zappatore, W. H. Fietz, R. Heller, L. Savoldi, M. J. Wolf, and R. Zanino, "A critical assessment of thermalhydraulic modeling of HTS twisted-stacked-tape cable conductors for fusion applications," Superconductor Science and Technology, vol. 32, no. 8, p. 084004, 2019, doi: 10.1088/1361-6668/ab20a9.

[13] R. Heller, P. Blanchier, W. H. Fietz, and M. J. Wolf, "Quench Analysis of the HTS CrossConductor for a Toroidal Field Coil," IEEE Trans. Appl. Supercond., vol. 29, no. 7, pp. 1-11, 2019, doi: 10.1109/tasc.2019.2917154.

[14] R. Kang, D. Uglietti, R. Wesche, K. Sedlak, P. Bruzzone, and Y. Song, "Quench Simulation of REBCO Cable-in-Conduit Conductor With Twisted Stacked-Tape Cable," IEEE Trans. Appl. Supercond., vol. 30, no. 1, pp. 1-7, 2020, doi: 10.1109/tasc.2019.2926258.

[15] C. Marinucci, L. Bottura, M. Calvi, and R. Wesche, "Quench Analysis of a High-Current Forced-Flow HTS
Conductor Model for Fusion Magnets," IEEE Trans. Appl. Supercond., Article vol. 21, no. 3, pp. 2445-2448, Jun 2011, doi: 10.1109/tasc.2010.2090329.

[16] L. Bottura, M. Calvi, and A. Siemko, "Stability analysis of the LHC cables," Cryogenics, vol. 46, no. 7-8, pp. 481-493, JulAug 2006, doi: 10.1016/j.cryogenics.2006.01.012.

[17] L. Bottura, C. Rosso, and M. Breschi, "A general model for thermal, hydraulic and electric analysis of superconducting cables," Cryogenics, vol. 40, no. 8-10, pp. 617-626, Aug-Oct 2000, doi: Doi 10.1016/S0011-2275(01)00019-4.

[18] "The SuperMagnet Suite." https://supermagnet.sourceforge.io/software.html (accessed.

[19] L. Bottura, C. Marinucci, and C. Rosso, "Two-channel analysis of QUELL experimental results," IEEE Trans. Appl. Supercond., vol. 10, no. 1, pp. 1106-1109, Mar 2000, doi: Doi 10.1109/77.828426.

[20] C. Marinucci, L. Bottura, G. Vecsey, and R. Zanino, "The QUELL experiment as a validation tool for the numerical code Gandalf," Cryogenics, vol. 38, no. 5, pp. 467-477, May 1998, doi: Doi 10.1016/S0011-2275(98)00014-9.

[21] S. Hahn et al., "Design Study on a 100-kA/20-K HTS Cable for Fusion Magnets," IEEE Trans. Appl. Supercond., vol. 25, no. 3, pp. 1-5, 2015, doi: 10.1109/tasc.2014.2371068.

[22] L. Bottura, "Stability, protection and ac loss of cable-inconduit conductors - a designer's approach," Fusion Eng. Des., vol. 20, pp. 351-362, 1993, doi: 10.1016/09203796(93)90065-p.

[23] P. Bruzzone, "30 years of conductors for fusion: A summary and perspectives," IEEE Trans. Appl. Supercond., Article; Proceedings Paper vol. 16, no. 2, pp. 839-844, Jun 2006, doi: 10.1109/tasc.2006.873342.

[24] N. Bykovsky, "HTS high current cable for fusion application," PhD, EPFL, 2017. 